\title{
Application of the PredictAD Software Tool to Predict Progression in Patients with Mild Cognitive Impairment
}

\author{
Anja H. Simonsen ${ }^{a} \quad$ Jussi Mattilab Anne-Mette Hejla Kristian S. Frederiksen ${ }^{a}$ \\ Sanna-Kaisa Herukkac, ${ }^{c}$ Merja Hallikainen ${ }^{c}$ Mark van Gils ${ }^{b}$ Jyrki Lötjönen ${ }^{b}$ \\ Hilkka Soininen ${ }^{c, d}$ Gunhild Waldemar ${ }^{a}$ for the Alzheimer's Disease \\ Neuroimaging Initiative \\ aMemory Disorders Research Group, Department of Neurology, Copenhagen University Hospital Rigshospitalet, \\ Copenhagen, Denmark; bVTT Technical Research Centre of Finland, Tampere, and CInstitute of Clinical Medicine- \\ Neurology, University of Eastern Finland, Yliopistoranta 1C and dDepartment of Neurology, Kuopio University \\ Hospital, Kuopio, Finland
}

\section{Key Words}

Mild cognitive impairment • Alzheimer's disease •

PredictAD software tool

\begin{abstract}
Background: The PredictAD tool integrates heterogeneous data such as imaging, cerebrospinal fluid biomarkers and results from neuropsychological tests for compact visualization in an interactive user interface. This study investigated whether the software tool could assist physicians in the early diagnosis of Alzheimer's disease. Methods: Baseline data from 140 patients with mild cognitive impairment were selected from the Alzheimer's Disease Neuroimaging Study. Three clinical raters classified patients into 6 categories of confidence in the prediction of early Alzheimer's disease, in 4 phases of incremental data presentation using the software tool. A 5th phase was done with all available patient data presented on paper charts. Classifications by the clinical raters were compared to the clinical diagnoses made by the
\end{abstract}

Alzheimer's Disease Neuroimaging Initiative investigators. Results: A statistical significant trend $(p<0.05)$ towards better classification accuracy (from 62.6 to $70.0 \%$ ) was found when using the PredictAD tool during the stepwise procedure. When the same data were presented on paper, classification accuracy of the raters dropped significantly from 70.0 to $63.2 \%$. Conclusion: Best classification accuracy was achieved by the clinical raters when using the tool for decision support, suggesting that the tool can add value in diagnostic classification when large amounts of heterogeneous data are presented.

Copyright $\odot 2012$ S. Karger AG, Basel

Data used in preparation of this article were obtained from the Alzheimer's Disease Neuroimaging Initiative (ADNI) database (adni. loni.ucla.edu). As such, the investigators within the ADNI contributed to the design and implementation of ADNI and/or provided data but did not participate in analysis or writing of this report. A complete listing of ADNI investigators can be found at: http://adni. loni.ucla.edu/wp-content/uploads/how_to_apply/ADNI_Acknowledgement_List.pdf.

\section{KARGER \\ Fax +4161306 1234}

E-Mail karger@karger.ch

www.karger.com
(C) 2012 S. Karger AG, Basel

$1420-8008 / 12 / 0346-0344 \$ 38.00 / 0$

Accessible online at:

www.karger.com/dem
Dr. Anja Hviid Simonsen

Memory Disorders Research Group

Department of Neurology; N6702

9, Blegdamsvej, DK-2100-Copenhagen (Denmark)

E-Mail anja.hviid.simonsen@rh.regionh.dk 


\section{Introduction}

Mild cognitive impairment (MCI) is a term referring to persons who do not fulfill the criteria for dementia, but who exhibit some form of cognitive impairment $[1,2]$. MCI is associated with an increased risk of developing Alzheimer's disease (AD) [3, 4]. Identification of MCI patients who will progress to $\mathrm{AD}$ would allow the application of disease-modifying treatments to slow progression at a point where clinical manifestations are limited. A combination of results from neuropsychological testing [5], MRI [6] and cerebrospinal fluid (CSF) biomarkers [7] can aid in the prediction of which patients with MCI will progress to AD. Furthermore, measurements of brain amyloid by PET using the ligand ${ }^{11} \mathrm{C}$ PIB (Pittsburg compound $\mathrm{B}$ ) were shown to predict a 3-year conversion to $\mathrm{AD}$ in a group of patients with amnestic MCI [8].

Even a modest delay of 1 year in the onset and progression of disease could drastically reduce the burden of AD on society [9]. Current symptomatic treatments and nonpharmacological interventions are assumed to be most effective at the earliest stages of the disease, underlining the importance of early diagnosis [10]. PredictAD, funded by the 7th EU framework (FP7 - 224328), is a research project where a consortium of technical and clinical partners aims to provide standardized and objective solutions for enabling earlier diagnoses of $\mathrm{AD}$, improved monitoring of treatment efficacy, easier patient selection for drug trials and improved cost-effectiveness of diagnostic protocols. For this purpose, the PredictAD consortium has developed a software tool to support clinical decisionmaking [11]. The PredictAD tool integrates heterogeneous data from clinical investigations in an individual patient, such as imaging, CSF biomarkers and results from neuropsychological tests for compact visualization in an interactive user interface. The aim of this study was to investigate whether the PredictAD tool could assist physicians in the early diagnosis of AD. The hypothesis was that when physicians were presented with clinical and paraclinical information from a patient by the software they would be able to predict conversion from MCI to $\mathrm{AD}$ better than if the information were presented in a traditional form on a printed chart.

\section{Methods}

\section{Patients}

Baseline data from 140 patients with MCI were selected from the Alzheimer's Disease Neuroimaging Initiative (ADNI) study cohort [4]. The ADNI comprises a consortium of universities and
Table 1. Average age, MMSE and years of education of the MCI patients

\begin{tabular}{llllll}
\hline & $\mathrm{n}$ & $\begin{array}{l}\text { Gender } \\
\text { M/F }\end{array}$ & $\begin{array}{l}\text { Age, years } \\
\text { (SD) }\end{array}$ & $\begin{array}{l}\text { MMSE } \\
(\mathrm{SD})^{*}\end{array}$ & $\begin{array}{l}\text { Years of educa- } \\
\text { tion (SD)* }\end{array}$ \\
\hline P-MCI & 64 & $39 / 25$ & $74.7(7.0)$ & $26.6(1.9)$ & $15.3(3.0)$ \\
S-MCI & 76 & $51 / 25$ & $73.9(7.8)$ & $27.3(1.7)$ & $16.3(2.7)$ \\
\hline
\end{tabular}

* Statistically significant difference $(\mathrm{p}<0.05$ from unpaired Student $t$ test).

medical centers in the USA and Canada. It was established to develop imaging techniques and biomarker procedures in normal subjects, subjects with MCI and subjects with mild AD. A total of 229 cognitively normal subjects, 398 MCI patients and 192 AD patients were recruited for the ADNI [4] which is supported by the National Institutes of Health, private pharmaceutical companies and nonprofit organizations. Full inclusion and exclusion criteria are described in detail at www.adni-info.org.

The criteria for selecting MCI patients in this study were: the availability of the Mini Mental State Examination (MMSE), Functional Assessment Questionnaire (FAQ), Alzheimer's Disease Assessment scale (ADAS-cog), Clinical Dementia Rating (CDR), CSF levels of amyloid beta and tau as well as MRI-derived volume values at the baseline measurement. Furthermore, patients who dropped out of the ADNI before 3 years of follow-up were excluded from our study. Some of the excluded patients had converted from MCI to AD at that time.

All selected patients had a CDR $=0.5$ and an MMSE score $\geq 24$ at baseline. In total, 140 of the 398 ADNI MCI patients met our criteria and were included in this study.

\section{Diagnostic Classification of Patients According to the ADNI}

In the ADNI study, patients with MCI were diagnosed according to the criteria of Petersen et al. [1]. Of the 140 patients included in our study, 64 (45.7\%) progressed to AD (progressive MCI, $\mathrm{P}-\mathrm{MCI}$ ) during the ADNI follow-up period according to the $\mathrm{Na}$ tional Institute of Neurological and Communicative Disorders and Stroke and Alzheimer's Disease and Related Disorders Association (NINCDS-ADRDA) [12], whereas 76 remained in the MCI group (stable MCI, S-MCI). Demographic information is listed in table 1 . Due to our selection criteria, the average time of conversion from MCI to AD (i.e. the P-MCI group) was 20 months, which is slightly longer than when considering all ADNI P-MCIs including the ones that were excluded because they dropped out before the end of the 3-year follow-up. The mean age of the patients was 74.3 years (SD 7.5), and there were 90 males and $50 \mathrm{fe}$ males.

Stepwise Classification by Clinical Raters Using the PredictAD Tool

In PredictAD, a clinical decision support tool was developed for the early diagnosis of AD [11]. The tool was applied by 3 clinical raters who were informed that the patients to be evaluated had been diagnosed with MCI by the ADNI team at baseline. All cases presented to the raters were anonymized. The baseline data 
Table 2. Protocol for stepwise data presentation using the PredictAD tool

Available data

Phase 1 Age, gender, years of education, primary occupation + MMSE including subscores, FAQ including subscores and $\mathrm{AD}$ index calculated by the software

Phase 2 All from phase $1+$ ADAS-cog including subscores and CDR including subscores

Phase 3 All from phases 1 and $2+$

MRI volumetrics (provided by the ADNI and computed with the FreeSurfer image analysis suite [14])

Phase 4 All from phases 1, 2 and $3+$ CSF levels of amyloid beta and total tau

were presented to the raters in a stepwise manner using the PredictAD tool. After each presentation of patient data, the clinical raters were asked to categorize the patient into one of 6 categories according to the likelihood that the patient would develop AD dementia: (1) clear indication of non-AD, (2) probable indication of non-AD, (3) subtle indication of non-AD, (4) subtle indication of early AD, (5) probable indication of early AD or (6) clear indication of early AD.

In other words, the clinical raters were asked to predict the 3-year conversion outcomes (S-MCI or P-MCI) using baseline data for cognitive tests, MRI and CSF biomarkers. The non-AD categories, i.e. (1)-(3), were defined to be used for subjects with memory problems thought to be due to causes other than earlyphase AD.

As shown in table 2, baseline clinical data were presented to the clinical raters in a stepwise manner in 4 phases, each phase adding more information about the patient.

In phase 1, only simple demographic and clinical data (global and functional status) were presented. In subsequent phases, more clinical information was added, mimicking the routine clinical diagnostic process, starting with the less costly and invasive procedures and ending with the addition of CSF biomarkers at phase 4 . The raters did not receive any form of feedback about their performance between the rating phases.

During each phase, the PredictAD tool provided only the allowed patient information to the clinical raters (see fig. 1). A timeline panel showed when the different tests were administered. Selecting a test from the timeline displayed it in a preview panel showing detailed results from the selected test. A tree of colored nodes on the right panel of the computer screen showed how patient data as a whole relate to average values from previously diagnosed S-MCI and P-MCI cases. Tests with large node sizes indicated patient measures which differentiated well between S-MCI and P-MCI. Red indicated patient data which are similar to P-MCI cases, whereas blue pointed towards S-MCI. White indicated measurements that were intermediate between the average values for previously diagnosed S-MCI and P-MCI cases. Values within the tree visualization are Disease State In- dex (DSI) values [13]. The DSI method is a novel statistical classification method that was developed with the goal of supporting clinical decision-making. Here, it was used for ranking patient data against data from known S-MCI and P-MCI cases on a scale of $0-1$. A DSI value of zero denotes a perfect match with S-MCIs, and a DSI value of 1 denotes a perfect match to P-MCI cases. To compute these DSI values, all MCI patients from the ADNI were used for training the disease model, except the patient being currently classified equivalent to leave-one-out cross-validation. This was done to ensure that there are enough data for good-quality computerized analysis and to ensure that raters' predictions have no bias towards the correct categories when using the software tool. More rigorous analysis of the performance and characteristics of the DSI method underlying the tool's visualizations can be found in our previously published paper [11].

To minimize influence from previous phases, all 140 patients were categorized once before moving on to the next phase and restarting from the first patient with additional clinical information. Information about previous rater categorizations was not provided to the clinical raters during subsequent phases. The presentation of the subjects was always in the same order. Any retention from previous phases influenced categorizations similarly, whether using the tool or reading the paper charts.

\section{Patient Classification by Clinical Raters Using Traditional Paper Charts}

After categorizing patients into 4 phases with the tool, each rater was presented with exactly the same patient data as at phase 4 in a paper chart format at a separate rater meeting. Raters were once more requested to classify all patients into the 6 categories. This 5th phase resembled the current state of diagnostic work, i.e. no help from tools was available, only raw data and test results. Similar to the earlier phases using the tool, the raters were not provided with test norms or MCI cut-off scores for any of the data.

\section{Clinical Raters}

The raters were all physicians who had clinical experience in the diagnostic evaluation of dementia and experience with the cognitive tests and assessment scales used in the ADNI. However, they had not previously participated in any ADNI-related studies.

\section{Statistical Analysis}

Categorizations made by the clinical raters using baseline data alone were compared to clinical diagnoses made by the ADNI investigators after 3 years of follow-up. At each phase, assigning a patient to category (1), (2) or (3) was deemed correct if the clinical diagnosis was S-MCI and similarly, categories (4), (5) and (6) were correct if the clinical diagnosis was P-MCI.

Classification accuracy for the raters was computed from all categorizations made by the 3 clinical raters grouped together. Classification accuracy was defined as the ratio of correctly classified patients versus the total number of patients.

Differences between the diagnostic groups at baseline were evaluated with the unpaired Student $t$ test. Changes in classification accuracy between the tool and the paper charts were evaluated using the McNemar test.

A subanalysis of cases categorized by the raters as (1) or (6) was performed separately. Differences in the relative number of clear 


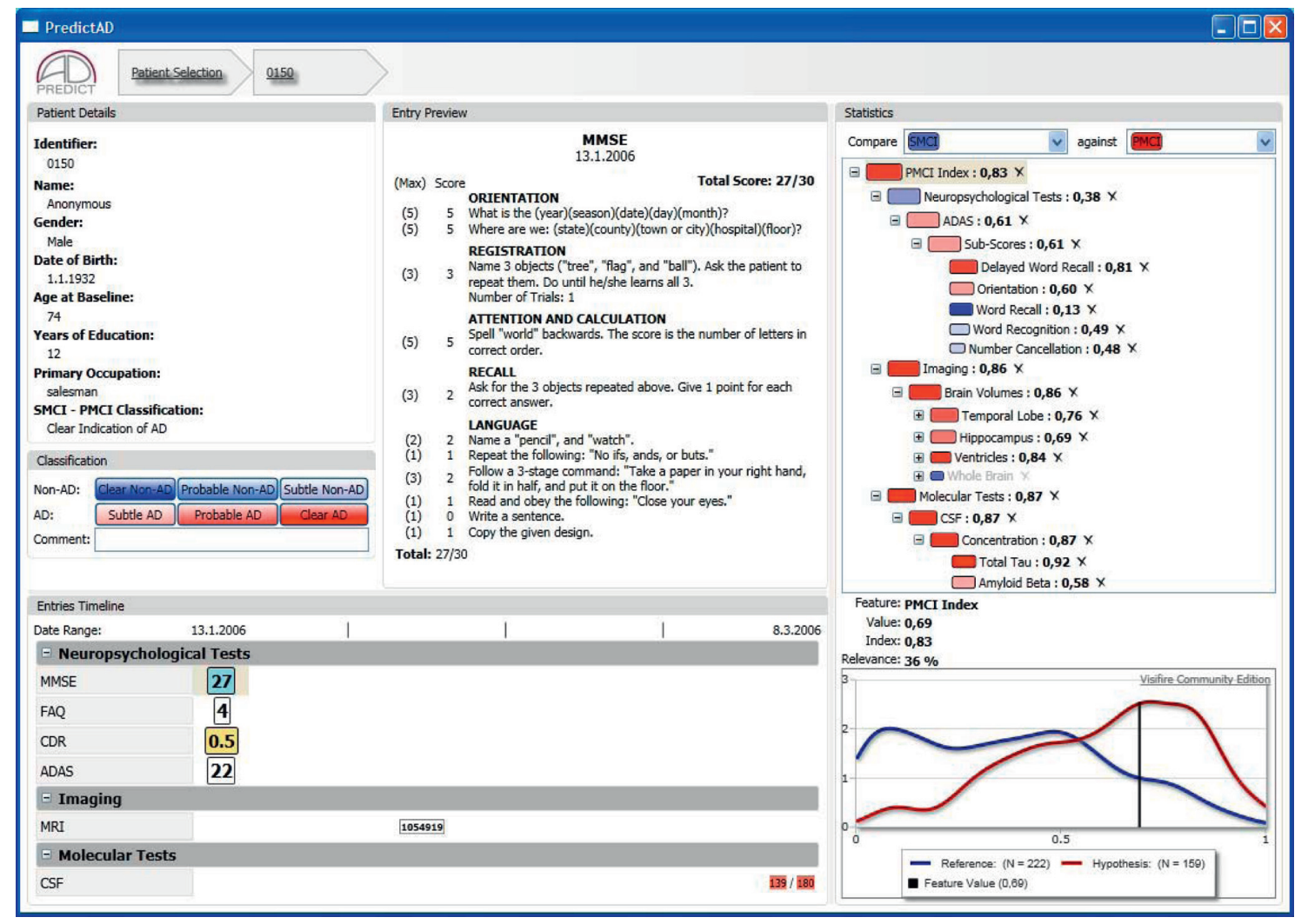

Fig. 1. Screenshot of the PredictAD tool used in this study. A patient being categorized at phase 4 has been marked as having a clear indication of early AD. Shown in the software are patient details, a timeline of entries where an MMSE entry is currently selected, a preview of the selected entry and a DSI visualization tree revealing how patient data relates to known S-MCI and P-MCI cases. Large nodes in the tree indicate good patient measures at differentiating between S-MCI and P-MCI. Shades of blue and red indicate where patient data are similar to S-MCIs and P-MCIs, respectively. Distributions (S-MCIs in blue, P-MCIs in red) and the patient value (in black) of the currently selected measure are displayed in a graph below the tree.

cases and in classification accuracy between phases were analyzed using the Fisher exact test.

Interrater agreement was evaluated using categorizations obtained at the 2 final phases where all data were available. This allowed us to test the agreement between clinical raters using the PredictAD software and paper charts. The test was performed by computing quadratic-weighted Cohen's kappa values.

In this study, a difference was considered statistically significant if $\mathrm{p}<0.05$.

\section{Results}

In this study, 140 patients with MCI at baseline were evaluated by 3 clinical raters. There were no differences between S-MCI and P-MCI regarding gender or age, but the S-MCI patients had a statistically significant higher
$\operatorname{MMSE}(\mathrm{p}=0.011)$ and more years of education $(\mathrm{p}=0.044)$ than the P-MCI patients (see table 1).

In total, the stepwise classification process resulted in 2,100 patient categorizations ( 140 patients $\times 3$ clinical raters $\times 5$ phases).

\section{Increasing Accuracy and Confidence from the} Availability of More Data

There was a trend towards more accurate classifications at each successive phase when the raters were presented with more clinical data, as depicted in figure 2 . The increase in accuracy from phase 1 to phase 4, i.e. $62.6-70.0 \%$, was statistically significant, $\mathrm{p}=0.029$.

At each successive phase, the raters gained more confidence in making the classifications. The number of patients assigned to the Clear categories increased by a sta- 
Fig. 2. Classification accuracy of clinical raters achieved for all patients during different phases of patient data presentation.
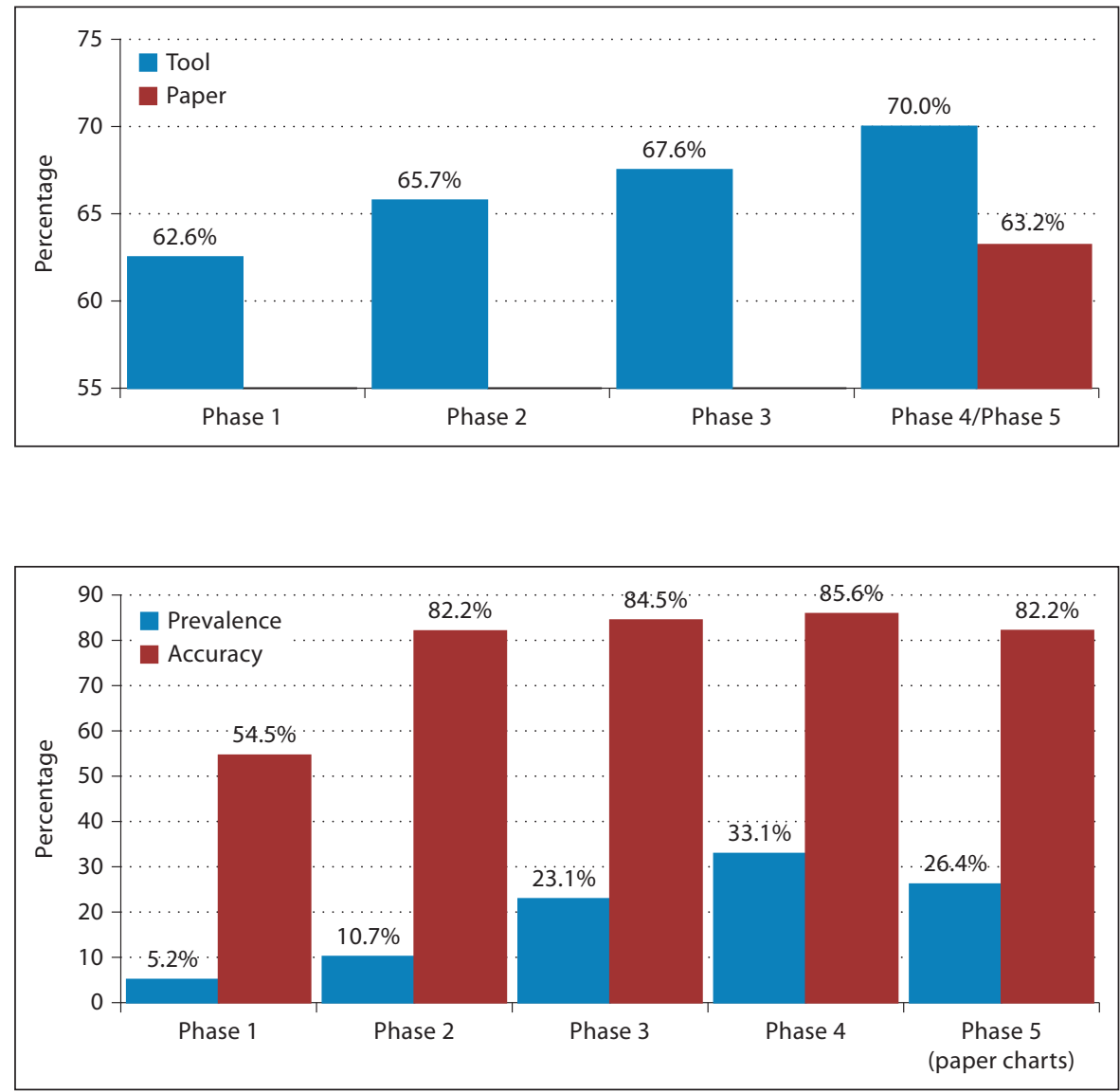

Fig. 3. Prevalence of patients assigned as clear (1) or (6) and classification accuracy for these cases at each phase. having it was close to being statistically significant ( $\mathrm{p}=$ 0.050). Classification accuracy of Clear cases was also lower with the paper charts than with the tool, but the difference was not statistically significant.

\section{Inter-Rater Agreement}

When the 3 raters were using the tool, inter-rater agreement between them was very good with Cohen's kappa of $0.64,0.76$ and 0.80 . When deprived of the tool, the agreement between raters was moderate (Cohen's kappa: $0.41,0.43$ and 0.71 ). Agreement between classifications made by a single rater using either the tool or paper charts was relatively good (Cohen's kappa: $0.58,0.70$ and 0.77).

\section{Discussion}

The results show that the best classification accuracy by clinical raters and the best agreement between raters was achieved when they used the soft ware tool for decision 
support. That is to say, when clinical raters combined their clinical experience in AD with the additional information and context provided by the tool, they achieved the most accurate and consistent results.

There was a statistically significant decrease in the classification accuracy of the raters when they only had traditional paper charts with patient data. This suggests it is more challenging to apply clinical diagnostic criteria to a large amount of heterogeneous data when they are presented without any help that highlights important details. These results reinforce the case for decision-support systems that help clinicians manage large quantities of patient data obtained in modern healthcare.

Another aspect revealed by the results was that increasing amounts of data proved beneficial for the diagnostics in more than one way. It was expected that the availability of more data would improve classification accuracy, but there was also an added benefit of boosting confidence in the diagnosis. There was a statistically significant increase in the number of clear cases each time more data were made available, up to $33.1 \%$ of the patients being marked clear at the 4 th phase. Classification accuracy of the clear cases also improved at each consecutive phase, up to $85.6 \%$. In other words, one third of the patients were classified from baseline data at a relatively high accuracy by the clinical raters. Although the overall prediction accuracy with the tool was only $70 \%$, having one third of the patients categorized at $85 \%$ accuracy (similar to the accuracy of clinical AD diagnoses) on average 20 months before the clinical AD diagnoses were given, could allow earlier treatments or better patient selection for drug trials. This result also suggests that there are some cases where the data contain strong evidence of early $\mathrm{AD}$, perhaps allowing earlier diagnosis if interpreted correctly. In this regard, having the tool seemed to improve classification performance, as with the paper charts, the doctors categorized only $26.0 \%$ of the cases as clear and achieved $82.2 \%$ classification accuracy for them.

To put this study into context, it is important to stress that the 3 clinical raters had not met the patients in person and they were not able to review the patients' medical history. By interviewing and examining patients in person, valuable information regarding medical history, psychiatric symptoms and information about the onset of cognitive decline would be obtained that could further improve the diagnostic classification of the patients. The tool used here should be developed further to take other clinical parameters into account such as the ones mentioned above.
The clinical raters in our study only had the baseline data presented to them by the software or on the paper charts. Whether the software solution would add clinically meaningful value to the classification accuracy above that obtained by interview and examination of patients in person together with traditional presentation of investigational results is not clear from this study. This hypothesis should be examined in further prospective clinical studies.

A weakness of this study is that some of the stable MCI patients may have converted to $\mathrm{AD}$ after the 3-year follow-up period. These patients were classified as S-MCI in the data, but may in fact have been P-MCIs. This may skew the results towards a lower classification accuracy, affecting all the methods and analyses applied in the study.

Furthermore, the ADNI MCI population was a selected group of patients as opposed to a general mixed memory-clinic population. The performance of the PredictAD tool in a mixed group of patients with memory impairment has still to be clarified.

In conclusion, the results suggest that a computerized decision-support tool designed to help the reading and interpreting of heterogeneous patient data may be useful for diagnostic work. When raters were using the tool, the confidence in making a diagnostic classification, the accuracy of the diagnoses and interrater agreement were all significantly higher than their performance when only traditional paper charts were available.

\section{Acknowledgements}

This study was sponsored by the 7th Framework Programme of the European Commission EU-Grant-224328-Predict-AD and the Simon Spies (G.W.) and Absalon foundations (G.W. and A.H.S.).

Data collection and sharing for this project was funded by the ADNI (National Institutes of Health grant U01 AG024904).

\section{Disclosure of Conflict of Interest}

J.M. has a patent application PCT/FI2010/050545 pending 2010. M.v.G. is an associate editor of the journal: Computer Methods and Programs in Biomedicine. J.L. has a patent (Disease State Index and fingerprint technology) patent US7,840,510B2 issued 2010 and a patent application PCT/FI2010/050545 pending 2010. G.W. is a board member of the Lundbeck Foundation and serves as a speaker/consultant for Pfizer, Janssen and Lundbeck. The remaining authors have no disclosures. 


\section{References}

$>1$ Petersen RC, Smith GE, Waring SC, Ivnik RJ, Tangalos EG, Kokmen E: Mild cognitive impairment: clinical characterization and outcome. Arch Neurol 1999;56:303-308.

$\checkmark 2$ Palmer K, Fratiglioni L, Winblad B: What is mild cognitive impairment? Variations in definitions and evolution of nondemented persons with cognitive impairment. Acta Neurol Scand 2003;197:14-20.

$>3$ Petersen RC, Negash S: Mild cognitive impairment: an overview. CNS Spectr 2008;13: 45-53.

4 Landau SM, Harvey D, Madison CM, Reiman EM, Foster NL, Aisen PS, Petersen RC, Shaw LM, Trojanowski JQ, Jack CR Jr, Weiner MW, Jagust WJ: Comparing predictors of conversion and decline in mild cognitive impairment. Neurology 2010;75:230-238.

5 Madureira S, Verdelho A, Moleiro C, Ferro JM, Erkinjuntti T, Jokinen H, Pantoni L, Fazekas F, Van der Flier W, Visser M, Waldemar G, Wallin A, Hennerici M, Inzitari D: Neuropsychological predictors of dementia in a three-year follow-up period: data from the LADIS study. Dement Geriatr Cogn Disord 2010;29:325-334.
6 Julkunen V, Niskanen E, Muehlboeck S, Pihlajamaki M, Kononen M, Hallikainen M, Kivipelto M, Tervo S, Vanninen R, Evans A, Soininen H: Cortical thickness analysis to detect progressive mild cognitive impairment: a reference to Alzheimer's disease. Dement Geriatr Cogn Disord 2009;28:404-412.

$>7$ Brandt C, Bahl JC, Heegaard NH, Waldemar G, Johannsen P: Usability of cerebrospinal fluid biomarkers in a tertiary memory clinic. Dement Geriatr Cogn Disord 2008;25: 553-558.

8 Koivunen J, Pirttila T, Kemppainen N, Aalto S, Herukka SK, Jauhianen AM, Hanninen T, Hallikainen M, Nagren K, Rinne JO, Soininen H: PET amyloid ligand [11C]PIB uptake and cerebrospinal fluid beta-amyloid in mild cognitive impairment. Dement Geriatr Cogn Disord 2008;26:378-383.

$\checkmark 9$ Brookmeyer R, Johnson E, Ziegler-Graham $\mathrm{K}$, Arrighi HM: Forecasting the global burden of Alzheimer's disease. Alzheimers Dement 2007;3:186-191.
10 Cummings JL: Treatment of Alzheimer's disease: the role of symptomatic agents in an era of disease-modifying therapies. Rev Neurol Dis 2007;4:57-62.

11 Mattila J, Koikkalainen J, Virkki A, Simonsen A, van Gils M, Waldemar G, Soininen H, Lotjonen J: A disease state fingerprint for evaluation of Alzheimer's disease. J Alzheimers Dis 2011;27:163-176.

12 McKhann G, Drachman D, Folstein M, Katzman R, Price D, Stadlan EM: Clinical diagnosis of Alzheimer's disease: report of the NINCDS-ADRDA Work Group under the auspices of Department of Health and $\mathrm{Hu}$ man Services Task Force on Alzheimer's Disease. Neurology 1984;34:939-944.

13 Chang C, Lin C: LIBSVM: a library for Support Vector Machines. Science 2001;2:1-39.

14 Fischl B, Salat DH, Busa E, Albert M, Dieterich $M$, Haselgrove $C$, van der Kouwe A, Killiany R, Kennedy D, Klaveness S, Montillo A, Makris N, Rosen B, Dale AM: Whole brain segmentation: automated labeling of neuroanatomical structures in the human brain. Neuron 2002;33:341-355. 